\title{
Diagnosis of infection in patients with left ventricular assist device: PET or SPECT?
}

\author{
Damien Legallois, $M D,{ }^{\mathrm{a}, \mathrm{b}}$ Alain Manrique, $\mathrm{MD}, \mathrm{PhD}^{\mathrm{a}, \mathrm{c}, \mathrm{d}}$ \\ a Normandie Univ, UNICAEN, Signalisation, électrophysiologie et imagerie des lésions \\ d'ischémie-reperfusion myocardique, FHU REMOD-VHF, Caen, France \\ b Department of Cardiology, CHU de Caen, Caen, France \\ c Department of Nuclear Medicine, CHU de Caen, Caen, France \\ d GIP Cyceron PET Center, Investigations chez l'Homme, Caen, France
}

Received May 4, 2018; accepted May 4, 2018

doi: $10.1007 /$ s12350-018-1324-6

\section{See related article, pp. 42-55}

Significant advances in heart failure (HF) treatment have resulted in a decrease in morbi-mortality in patients with reduced left ventricular ejection fraction. Despite optimal treatment, some patients suffer from functional limitations due to a high symptom burden of shortness of breath, congestion and fatigue, and still have a decreased life expectancy. In this context, heart transplantation is considered as the therapy of choice for younger people who are free from significant comorbidity. ${ }^{1}$ However, most of these patients could not receive a heart transplant because of the shortage of matching donors. As a consequence, a growing number of patients with endstage $\mathrm{HF}$ and a high risk of short-term mortality undergo left ventricular assist device (LVAD) implantation either as an alternative to heart transplantation (i.e. destination therapy) or as a bridge to transplantation. The number of patients undergoing LVAD implantation increases every year, reaching in 2015 more than 3000 patients in the US. $^{1}$

All current pumps (whether axial or centrifugal) require an external power source with energy supplied via a tunneled percutaneous driveline. The latter also allows to control and communicate with the device

Reprint requests: Damien Legallois, MD, Normandie Univ, UNICAEN, Signalisation, électrophysiologie et imagerie des lésions d'ischémie-reperfusion myocardique, FHU REMOD-VHF, 14000,

Caen, France; damien.legallois@unicaen.fr

J Nucl Cardiol 2019;26:56-8.

$1071-3581 / \$ 34.00$

Copyright (C) 2018 American Society of Nuclear Cardiology. using an external controller. An abdominal driveline is the most common setting (HeartMate II, HeartMate III and HVAD). Patients with Jarvik 2000 device usually got a skull-pedestal for the power cable skin exit located in the retro-auricular region. The cable is then tunneled to the pump. Depending of the device, the pump is located in a pump pocket, which could be intra-pericardial, external to the pericardium, or partially inside. Whatever the type of pump, it sucks blood from the left ventricle by an inflow cannula within the left ventricle. The outflow graft of the LVAD is anastomosed to either the ascending or descending portion of the aorta, depending on the model of the LVAD. Because of the presence of the pump, it could be possible that the LVAD change the shape of the ventricle. In order to avoid thrombosis and stroke, patients have to be treated with vitamin-K antagonist and mean blood pressure must be cautiously monitored. Finally, current devices are continuous-flow and, as a result, it may not be possible to feel pulse or to measure blood pressure with a cuff.

LVADs led to a significant improvement in survival, functional capacity, and quality of life in patients with end-stage HF. However, this therapy is associated with a significant risk of complications related to the device itself (pump malfunction), to the frailty of the heart (mainly right ventricular failure due to right ventricular volume overload) or to the consequences of pump-patient interface including infection, thrombosis, and stroke. ${ }^{2}$ Among these, infectious complications are a particular concern as $20-40 \%$ of LVAD-recipients will develop sepsis within 2 years after implantation, ${ }^{2,3}$ especially since LVADs have expanded from short-term use as a bridge to transplantation to also include longterm destination therapy in patients who are not eligible for transplantation. ${ }^{4}$ Infections are usually classified as 
VAD-specific infections, VAD-related infections, and non-VAD infections in LVAD-recipients. ${ }^{5}$ VAD-specific infections are related to the hardware (e.g., pump and cannula infection, pocket infection, or percutaneous driveline infection) whereas VAD-related infections refer to those that can also occur in patients who do not have VADs (e.g., infective endocarditis, blood stream infection, or mediastinitis).

LVAD driveline infections are the most common type of LVAD-specific infections ${ }^{6}$ and can progress to deep tissue infections over time. ${ }^{7,8}$ Several factors contribute to driveline infection. First, the driveline exit site is a perfect conduit for bacteria entry of and the prosthetic material of the driveline itself is favorable to the formation of bacterial biofilms. ${ }^{9}$ Second, the mobility of the percutaneous driveline can lead to repetitive local trauma that further facilitates bacterial infection. The need for long-term antimicrobial therapy in these cases may lead to the emergence of drug-resistant organisms such as Pseudomonas and Staphylococcus aureus. As the removal of the device is usually not an option in most cases, LVAD infections are a devastating complication for affected patients and are associated with an increased rate of rehospitalization and mortality. 8,10 Consequently, infection is a leading cause of late mortality, with an estimated rate of approximately $20 \% 12$ months after the initial diagnosis of driveline infection. ${ }^{2,11}$ Gordon and al. showed a $22 \%$ overall infection rate of LVADs and a 1 year mortality 5.6 times greater in patients with infection compared to patients without LVAD infection. ${ }^{12}$ A recent study showed that $50 \%$ of infected LVAD-recipients died during a mean follow-up of 23 months after the diagnosis of infection using 18FFDG PET/CT. The existence of a central infection (pump and/or cannula) is a factor of worse prognosis. ${ }^{3}$ LVAD infections are also associated with an increased risk of including pump thrombosis, stroke, bleeding, longer hospital stay, need for LVAD exchange, and failure to transplant. ${ }^{13,14}$ However, infected patients can successfully benefit from heart transplantation, with a favorable outcome compared to a larger group of transplant recipients. ${ }^{10,15}$

Therefore, the diagnosis of device-related infection in patients with LVAD is a key issue in patients' management, and still remains challenging. Local infection signs (i.e., erythema or external suppuration) on physical examination have a low diagnostic value, and blood culture is usually poorly informative once antibiotics have been initiated. The use of computed tomography (CT), limited by metal device artifacts, yields nonspecific findings, and echocardiography is not useful in this clinical setting.

There is growing evidence that the use of $18 \mathrm{~F}-\mathrm{FDG}$ PET/CT improves the diagnosis of LVAD-related infection. ${ }^{3,16-18}$ In this issue, de Vaugelade et al. ${ }^{19}$ retrospectively analyzed the diagnostic performance of both radiolabeled leucocyte scintigraphy and 18F-FDG PET/CT in 24 LVAD-recipients with suspected devicerelated infection. Their conclusion is that $18 \mathrm{~F}-\mathrm{FDG}$ PET/CT demonstrated a higher sensitivity compared to radiolabeled leucocyte scintigraphy and could be preferred as first-line radionuclide imaging procedure. It is important to note that to achieve this high sensitivity of $95 \%$, the performance and interpretation of $18 \mathrm{~F}-\mathrm{FDG}$ PET/CT scans have to fulfill some technical requirements. First, the patients were submitted to a high-fat and low-carbohydrate diet the day before 18F-FDG PET imaging and were asked to fast for at least $12 \mathrm{~h}$ prior to examination. The observance of this diet, which is easier to achieve during hospitalization, is of paramount importance to suppress physiological myocardial FDG uptake. After only a $6 \mathrm{~h}$ fasting without additional diet, Dell' Aquila et al. ${ }^{17}$ reported a poor discriminator power of 18F-FDG PET/CT for pump housing infection, although the diagnostic value for the infection of the piercing site of driveline infection or its intracorporeal course was excellent. Second, the interpretation criteria must be clearly defined. In patients who recently underwent cardiac surgery, a postoperative inflammatory response may result in non-specific $18 \mathrm{~F}-\mathrm{FDG}$ uptake in the immediate postoperative period. While interpreting 18F-FDG PET/CT images, we have to keep in mind that normal uptake could have different patterns regarding to the material composition. Keidar and al. ${ }^{20}$ previously demonstrated that diffuse $18 \mathrm{~F}$-FDG uptake is frequently observed in non-infected vascular prostheses due to a foreign-body chronic low-grade inflammation. This latter phenomenon is more frequent with Dacron (that is present mostly in LVADs outflow) and should therefore be recognized as a cause of potential interpretation pitfall. As a result, Vaugelade et al. considered that a circumferential and homogeneous 18F-FDG uptake was a negative pattern for infection, defining positivity as a focal 18F-FDG uptake observed on both attenuation-corrected and uncorrected images in order to avoid overcorrection artifacts. ${ }^{19}$ Although previous findings emphasized the impact of quantitative analysis, ${ }^{21}$ Vaugelade et al. found no difference between visual and quantitative analysis of 18F-FDG PET/CT for the diagnosis of LVAD-related infection.

In addition, the authors reported a reduced sensitivity of radiolabeled leucocyte scintigraphy for the diagnosis of LVAD-related infection, related to a high rate of false negative examinations, most likely due to the early initiation of antibiotherapy. Radiolabeled leucocyte scintigraphy has been successfully used for cardiac implantable electronic device infections, especially when performed before the initiation of antibiotic 
therapy. ${ }^{22}$ However, its role for LVAD infections has not been clearly established. ${ }^{23}$

The results presented in this issue are a step forward in the demonstration of the accuracy of 18F-FDG PET/ CT for the early diagnosis of infection in patients with LVAD. However, it is very likely that the dissemination of this technique would benefit from a standardization of acquisition conditions and image interpretation.

\section{References}

1. Yancy CW, Jessup M, Bozkurt B, Butler J, Casey DE Jr, Drazner $\mathrm{MH}$, et al. 2013 ACCF/AHA guideline for the management of heart failure: A report of the American College of Cardiology Foundation/American Heart. J Am Coll Cardiol. 2013;62:e147239.

2. Kirklin JK, Naftel DC, Pagani FD, Kormos RL, Stevenson LW, Blume ED, et al. Seventh INTERMACS annual report: 15,000 patients and counting. J Heart Lung Transpl. 2015;34:1495-504.

3. Kim J, Feller ED, Chen W, Liang Y, Dilsizian V. FDG PET/CT for early detection and localization of left ventricular assist device infection: Impact on patient management and outcome. JACC Cardiovasc Imaging. 2018. https://doi.org/10.1016/j.jcmg.2018. 01.024 .

4. Wickline SA, Fischer KC. Can infections be imaged in implanted devices? ASAIO J. 2000;46:S80-1.

5. Hannan MM, Husain S, Mattner F, Danziger-Isakov L, Drew RJ, Corey GR, et al. Working formulation for the standardization of definitions of infections in patients using ventricular assist devices. J Heart Lung Transpl. 2011;30:375-84.

6. Leuck AM. Left ventricular assist device driveline infections: Recent advances and future goals. J Thorac Dis. 2015;7:2151-7.

7. Koval CE, Thuita L, Moazami N, Blackstone E. Evolution and impact of drive-line infection in a large cohort of continuous flow ventricular assist device recipients. J Heart Lung Transplant. 2014;33:1164-72.

8. Goldstein DJ, Naftel D, Holman W, Bellumkonda L, Pamboukian SV, Pagani FD, et al. Continuous-flow devices and percutaneous site infections: Clinical outcomes. J Heart Lung Transplant. 2012;31:1151-7.

9. Pereda D, Conte JV. Left ventricular assist device driveline infections. Cardiol Clin. 2011;29:515-27.

10. Smedira NG, Hoercher KJ, Lima B, Mountis MM, Starling RC, Thuita L, Schmuhl DM, Blackstone EH. Unplanned hospital readmissions after HeartMate II implantation: Frequency, risk factors, and impact on resource use and survival. JACC Heart Fail. 2013;1:31-9.

11. John R, Aaronson KD, Pae WE, Acker MA, Hathaway DR, Najarian KB, et al., for the HeartWare Bridge to Transplant ADVANCE Trial Investigators. Drive-line infections and sepsis in patients receiving the HVAD system as a left ventricular assist device. J Heart Lung Transplant 2014;33:1066-73

12. Gordon RJ, Weinberg AD, Pagani FD, Slaughter MS, Pappas PS, Naka Y, et al. Prospective, multicenter study of ventricular assist device infections. Circulation. 2013;127:691-702.

13. Trachtenberg BH, Cordero-Reyes AM, Aldeiri M, Alvarez $\mathrm{P}$, Bhimaraj A, Ashrith G, et al. Persistent blood stream infection in patients supported with a continuous-flow left ventricular assist device is associated with an increased risk of cerebrovascular accidents. J Card Fail. 2015;21:119-25.

14. Kilic A. The future of left ventricular assist devices. J Thorac Dis. 2015;7:2188-93.

15. Toda K, Yonemoto Y, Fujita T, Shimahara Y, Sato S, Nakatani T, et al. Risk analysis of bloodstream infection during long-term left ventricular assist device support. Ann Thorac Surg. 2012;94:138793.

16. Bernhardt AM, Pamirsad MA, Brand C, Reichart D, Tienken M, Barten MJ, et al. The value of fluorine-18 deoxyglucose positron emission tomography scans in patients with ventricular assist device specific infections. Eur J Cardiothorac Surg. 2017;51:10727.

17. Dell'Aquila AM, Avramovic N, Mastrobuoni S, Motekallemi A, Wisniewski K, Scherer M, et al. Fluorine-18 fluorodeoxyglucose positron emission tomography/computed tomography for improving diagnosis of infection in patients on CF-LVAD: Longing for more 'insights'. Eur Heart J Cardiovasc Imaging. 2017. https://doi.org/10.1093/ehjci/jex158.

18. Kanapinn P, Burchert W, Körperich H, Körfer J. 18F-FDG PET/ CT-imaging of left ventricular assist device infection: A retrospective quantitative intrapatient analysis. J Nucl Cardiol. 2018. https://doi.org/10.1007/s12350-017-1161-z.

19. de Vaugelade C, Mesguich C, Nubret K, Camou F, Greib C, Dournes $\mathrm{G}$, et al. Infections in patients using ventricular assist devices: Comparison of the diagnostic performance of 18F-FDG PET/CT scan and leucocyte labeled scintigraphy. J Nucl Cardiol 2018;in press.

20. Keidar Z, Pirmisashvili N, Leiderman M, Nitecki S, Israel O. 18FFDG uptake in noninfected prosthetic vascular grafts: Incidence, patterns, and changes over time. J Nucl Med. 2014;55:392-5.

21. Avramovic N, Dell'Aquila AM, Weckesser M, Milankovic D, Vrachimis A, Sindermann JR, et al. Metabolic volume performs better than SUVmax in the detection of left ventricular assist device driveline infection. Eur $\mathrm{J}$ Nucl Med Mol Imaging. 2017;44:1870-7.

22. Erba PA, Sollini M, Conti U, Bandera F, Tascini C, de Tommasi $\mathrm{SM}$, et al. Radiolabelled WBC scintigraphy in the diagnostic workup of patients with suspected device-related infections. JACC Cardiovasc Imaging. 2013;6:1075-86.

23. Litzler PY, Manrique A, Etienne M, Salles A, Edet-Sanson A, Vera P, et al. Leukocyte SPECT/CT for detecting infection of leftventricular-assist devices: Preliminary results. J Nucl Med. 2010;51:1044-8. 